\title{
Effect of Supplemental Signal on Start-Up Lost Time at Signalized Intersection
}

\author{
Yongfeng Ma and Jian Lu \\ Jiangsu Key Laboratory of Urban ITS, Southeast University, Si Pai Lou No.2, Nanjing 210096, China
}

\begin{abstract}
Supplemental signals have been widely used at intersections to reduce the impact of heavy vehicles. This study aims to measure the effects of supplemental signals on Start-Up Lost Time (SULT). A corrected method of calculating SULT considering heavy vehicles are presented. The method also introduce into basic saturation headways, locations of the heavy vehicles and the four types of headways. Field studies were implemented at 18 approaches of 6 intersections in Nanjing, China. The results show that the existence of heavy vehicles has significant influence on both of headways and SULT at signalized intersection. Supplemental signal is effective countermeasure to reduce the influence of heavy vehicles. The study will be helpful when measuring SULT at intersection with supplemental signal.
\end{abstract}

\section{Introduction}

As a critical node, signalized intersection plays an important role in the whole road network. There are much more vehicles driving into and crowded around signalized intersections, especially during the peak period, which lead to further poor performance of the whole road network. More and more heavy vehicles, containing buses or single unit trucks, also contribute to the phenomena from two aspects: (a) slow-start factor. Compared with cars, heavy vehicles start more slowly during green time. A heavy vehicle among the first few queuing vehicles will significantly increase the Start-Up Lost Time (SULT), which results in low traffic capacity; (b) block factor. Heavy vehicles will block views of the subsequent queuing vehicles (especially for cars) due to different height of vehicles, which will prevent related drivers from seeing and reacting to the change of the signals timely at the intersections. Then this slow reaction will also increase the SULT and lessen the traffic capacity. According to our survey, the proportion of heavy vehicles around signalized intersections has already reached $15 \% \sim 30 \%$, which is 5 to 10 times more than the default proportion 3\% assumed in the Highway Capacity Manual 2010 [1]. This high level of heavy vehicles makes an important impact on SULT and the traffic capacity. Therefore, how to reduce the influences of heavy vehicles at signalized intersection and the measurement of effectiveness of related countermeasures are very important and meaningful.

Many researchers are focusing on developing different countermeasures to improve the performance of signalized intersections. For the former factor, it is difficult to be eliminated, due to the heavy vehicles' design. But for the latter factor, much more countermeasures are put forward and one of which is to install the supplemental signals. In Manual on Uniform Traffic Control Devices for Streets and Highways (MUTCD, Edition 2009) [2], it is indicated that "Supplemental signal face is not a primary signal face but which is provided for a given approach or separate turning movement to enhance visibility or conspicuity". In recent years, to reduce the block factor at signalized intersections, supplemental signals have been increasingly deployed in urban areas in China. However, there are still very little publications or data to demonstrate the effectiveness of supplemental signals.

The calculation of SULT under different traffic environment is very important and also attracts many related researchers. Generally, the SULT can be derived by measuring the elapsed time for the first four vehicles, including the start-up response time (SRT) of the first vehicle, and adjust it with the average saturation headway [3]. The Highway Capacity Manual (HCM) is an important publication and keeps update of SULT and saturation headway in last twenty years. In edition 1994, the saturation flow rate is recommended as $1,800 \mathrm{pc} / \mathrm{hr} / \mathrm{ln}$, which corresponds to a saturation headway of $2 \mathrm{sec}$. In edition 1997 and 2000, the saturation flow rate is increased to $1,900 \mathrm{pc} / \mathrm{hr} / \mathrm{ln}$, which corresponds to a saturation headway of $1.895 \mathrm{sec}$ [4]. And also, in edition 2000, SULT ranges from $1.0 \mathrm{sec}$ to $2.0 \mathrm{sec}$ based on typical observation. But the result is limited to the through movement. In the latest edition (edition 2010), the SULT is recommended as $2.0 \mathrm{sec}$ [1]. It also presents that the elapsed times of the first four vehicles in a queue are usually to estimate SULT and the average headway of the latter vehicles in a queue are usually recommended as 
saturation headway. Some other researchers also measure SULT and saturation flow rate with none or less heavy vehicles through field study [5]-[8].

With the development of economy, more and more heavy vehicles are on urban arterials [9]. A survey about motor vehicle use between 1972 and 2003 also reports that heavy vehicles make up more than $10 \%$ and are still increasing [10]. Many publications also pay their attentions on the disadvantage and propose some countermeasures. In HCM (edition 2010), a coefficient of equivalent is introduced to differ the passenger car and heavy vehicles. More and more studies [11]-[15] are focusing on effects of heavy vehicles. Rafai Alyami et al. examined the effects of truck percentages on SULT at a signalized intersection [9]. A new model is proposed to calculate SULT under mixed traffic. According to the field observations, the SULT is generally higher when trucks are in a queue and reach the highest when trucks are among the first four vehicles of a queue.

The primary objective of this study is to evaluate the effects of supplemental signals on SULT when heavy vehicles exist in a queue. More specifically, the study includes the following tasks: (1) Measuring the impacts of heavy vehicles on SULT at signalized intersections; (2) Determining whether supplemental signals can reduce the block effects of heavy vehicles at signalized intersections.

\section{Methodology}

Traditional calculation of SULT involves saturation headways. However, if we use saturation headways to calculate the SULT, it is often negative when heavy vehicles exist in the queue. In HCM (edition 2010), any headway affected by a heavy vehicle is not ideal, in other words, only headways that occur before the first vehicle's arrival can be treated as "ideal". However, under real traffic and existing method of data collection, many other factors affect the headways and their measurement, such as drivers, vehicles and traffic environment. Thus, in this study, the observed saturation headway is defined as basic saturation headways.

Generally, the first four headways of each cycle are discarded, which are taken as unstable. Thus, for each cycle, headways from the 5th vehicle to the one followed by a heavy vehicle are included to calculate the basic saturation headway. The basic saturation headway can be calculated using the following equation:

$$
h=\frac{\sum_{i=1}^{n} \sum_{j=5}^{k_{i}} h_{j}^{\prime}}{\sum_{i=1}^{n}\left(k_{i}-4\right)}
$$

where,

$h$ - the basic saturation headway;

$h_{j}^{\prime}$-the headway of the $j$ th vehicle before the heavy one;

$k_{i}$-the number of vehicles in each cycle which are involved in this calculation; and

$n$ - the total number of cycles observed.

$$
l_{s}=\frac{\sum_{i=1}^{n^{\prime}} \sum_{j=1}^{4}\left(h_{j}-h\right)}{n^{\prime}}
$$

where,

$l_{s}$ - SULT;

$h_{j}-$ the headway of the $j$ th vehicle in the queue of ach cycle; and

$n-$ the number of cycles.

In order to distinguish the influences brought by the different locations of heavy vehicles in a queue, all observed signal cycles are divided into four categories before the calculation of SULT. The classification is shown as below:

Case 1: one or more heavy vehicles are among the first four vehicles at the red light, but none among the latter vehicles;

Case 2: one or more heavy vehicles are among the $5^{\text {th }}$ vehicle and followed vehicles at the red light, but none among the first four vehicles;

Case 3: one or more heavy vehicles are both among the first four vehicles and the latter vehicles; and

Case 4: no heavy vehicles were among a queue at the red light.

SULT for each case is calculated separately and then multiplied by their corresponding percentage of each case to obtain an overall SULT.

$$
l_{s}^{\prime}=\sum_{i=1}^{4} l_{i} \times p_{i}
$$

where,

$l_{s}^{\prime}-$ the corrected SULT;

$l_{i}-$ the SULT of each case; and

$p_{i}-$ the percent of each case in a period of time.

\section{Data collection}

There are two basic kinds of deployment for supplemental signals. Scenario 1: supplemental signals for left-turning vehicles are installed at the left side of the approach, and those for through and right-turning vehicles are installed above the approach. Scenario 2: supplemental signals for left-turning vehicles are installed at the left side of the approach and those for right-turning and through vehicles are installed at the right side of the approach.

Field data collection was conducted at 18 approaches at 6 signalized intersections. The selected sites are all located on arterials in Nanjing, China. All of the intersections are under count-down traffic signal control. The traffic environments of these intersections are similar. For each intersection, at least two approaches (one for left-turning and one for through) are equipped with supplemental signals, while only primary signals for other approaches. Since vehicles can turn right during the red phase at the intersections, the study only focused on left-turning and through vehicles. The following principles were used in selecting sites and field study:

- The selected sites should have enough left-turning and through vehicles to queue up on their lanes;

- There are no parking and bus stops adjacent to the intersection (about $250 \mathrm{ft}$. upstream or downstream of the stop line);

- Each approach of the intersection has exclusive lanes for left-turning and through vehicles; 
- Traffic conditions are steady during field study;

- No major pedestrian and non-motor activity; and

- Cycles with very slowly moving vehicles for some unusual condition (such as traffic congestion and traffic incident) were not included.

A typical layout of selected signalized intersections is illustrated in Fig. 1. Two video cameras were deployed on the top of a building near the intersection to record traffic. Data were collected during the AM peak hours (7:00 9:30a.m.).

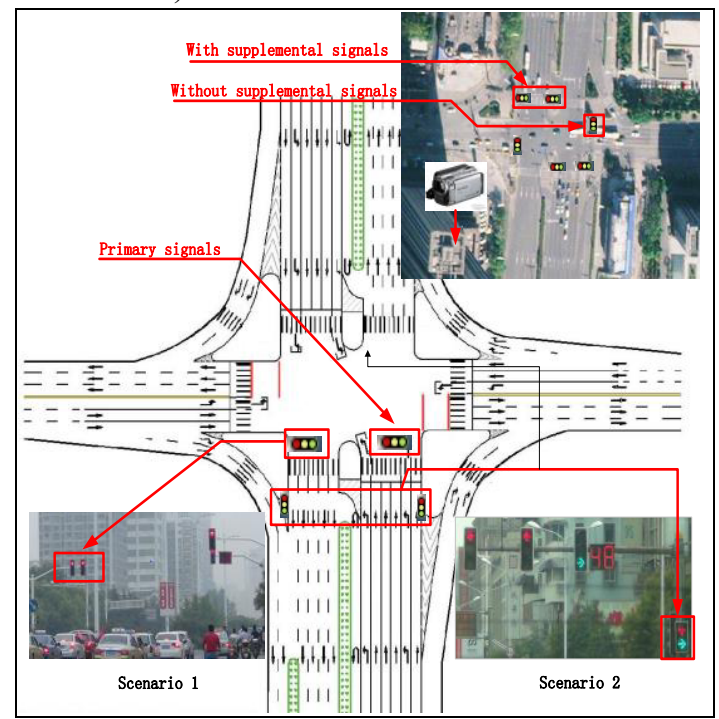

Figure 1. Sketch of supplemental signals and data collection.

Headways are generally measured at STOP line of approaches, and the reference point is generally the crossing of the rear axle of a vehicle over a STOP line. The first headway is recommended as the time from the initiation of the green indication to the crossing of the rear axle of the first vehicle over the STOP line. Only those vehicles that have come to a complete stop before the initiation of green indication are included. To better differ the heavy vehicles and passenger cars, four different types of headways were defined in this study [16], as follows:

- Type 1: (H-NH) the headways between a heavy vehicle and a car;

- Type 2: $(\mathrm{H}-\mathrm{H})$ the headways between two heavy vehicles;

- Type 3: (NH-NH) the headways between two cars;

- Type 4: (NH-H) the headways between a car and a heavy vehicle.

In total, 419 cycles for through movement and 285 cycles for left-turning movement were obtained. When the condition is "with supplemental signals", the queuing vehicles are affected only by the slow-start factor of heavy vehicles. Otherwise, the queuing vehicles are influenced by the slow-start factor and block factor of heavy vehicles.

\section{Data analysis}

\subsection{Saturation headways}

Since the first four headways in each cycle are affected by SULT, only the headways after the fourth vehicle were measured. A total of 2775 headways were obtained, including 970 left-turning headways and 1805 through headways. The summary for headway data is presented in Table 2 and Fig. 2. The mean saturation headways of leftturning vehicles vary from $1.30 \mathrm{~s}$ to $2.24 \mathrm{~s}$ with supplemental signals, and from $1.42 \mathrm{~s}$ to $2.42 \mathrm{~s}$ without supplemental signals. The mean saturation headways of through vehicles vary from $1.41 \mathrm{~s}$ to $2.13 \mathrm{~s}$ with supplemental signals, and from $1.44 \mathrm{~s}$ to $2.53 \mathrm{~s}$ without supplemental signals.

T-test was applied to identify whether saturation headways with supplemental signals were significantly different from that without supplemental signals. Most results, as shown in Table 2 were found to be significantly different statistically. However, there was no significantly difference for left turning and through vehicles in type 3 , and through vehicles in type 4 . It may be caused by the following two reasons: (a) cars do not have block effects on their following vehicles. So there is little difference in saturation headways for vehicles in type 3 and type 4, even after installing supplemental signals; (b) insufficient data were obtained when the approaches were controlled only by primary signals. The huge difference in number of samples may influent on the results of T-test. From Table 2, the mean saturation headways were decreased after installing supplemental signals, except for left turning vehicles in type 4. Furthermore, the mean saturation headways for vehicles in type 1 and type 3 are generally less than that for vehicles in type 2 and type 4 , which may be affected by the slow-start factor of heavy vehicles.

Table 1. Summary statistics of saturation headways (seconds).

\begin{tabular}{|c|c|c|c|c|c|c|c|c|}
\hline Type & Direction & Signal & $\mathrm{N}$ & Mean & Min. & Max. & $\begin{array}{l}\text { Std. } \\
\text { Dev. }\end{array}$ & $\mathrm{p}$-value \\
\hline \multirow{4}{*}{1} & \multirow{2}{*}{$\mathrm{L}$} & with & 55 & 1.30 & 0.84 & 2.24 & 0.32 & \multirow{2}{*}{0.007} \\
\hline & & N/A & 66 & 1.52 & 0.80 & 3.48 & 0.55 & \\
\hline & \multirow{2}{*}{$\mathrm{T}$} & with & 126 & 1.41 & 0.74 & 2.32 & 0.38 & \multirow{2}{*}{0.044} \\
\hline & & N/A & 37 & 1.55 & 1.15 & 2.48 & 0.32 & \\
\hline \multirow{4}{*}{2} & \multirow{2}{*}{$\mathrm{L}$} & with & 25 & 2.00 & 1.01 & 2.79 & 0.47 & \multirow{2}{*}{0.026} \\
\hline & & $\mathrm{N} / \mathrm{A}$ & 25 & 2.42 & 1.57 & 3.40 & 0.50 & \\
\hline & & with & 64 & 2.13 & 0.80 & 3.28 & 0.55 & \multirow{2}{*}{0.040} \\
\hline & & $\mathrm{N} / \mathrm{A}$ & 30 & 2.53 & 1.41 & 3.89 & 0.76 & \\
\hline \multirow{4}{*}{3} & & with & 196 & 1.36 & 0.66 & 2.78 & 0.34 & \multirow{2}{*}{0.062} \\
\hline & & N/A & 484 & 1.42 & 0.73 & 2.93 & 0.36 & \\
\hline & & with & 1142 & 1.42 & 0.58 & 3.20 & 0.39 & \multirow{2}{*}{0.452} \\
\hline & & $\mathrm{N} / \mathrm{A}$ & 281 & 1.44 & 0.59 & 3.17 & 0.41 & \\
\hline \multirow{4}{*}{4} & & with & 52 & 2.24 & 0.93 & 3.31 & 0.50 & \multirow{2}{*}{0.001} \\
\hline & & N/A & 67 & 1.89 & 0.89 & 3.15 & 0.60 & \\
\hline & & with & 93 & 2.13 & 0.66 & 3.43 & 0.59 & \multirow{2}{*}{0.811} \\
\hline & & $\mathrm{N} / \mathrm{A}$ & 32 & 2.10 & 1.02 & 3.30 & 0.52 & \\
\hline
\end{tabular}

\subsection{Start-up lost time (SULT)}

The cycles only with cars in type 3 are selected to calculate basic saturation headways. The results of basic saturation headways and saturation headways are presented in Fig. 3. As shown in Fig. 3, the basic saturation headways are $1.36 \mathrm{~s}$ and $1.42 \mathrm{~s}$ for left turning and through vehicles with supplemental signals respectively. However, for left turning and through 
vehicles without supplemental signals, the basic saturation headways, $1.42 \mathrm{~s}$ and $1.44 \mathrm{~s}$ respectively, are little bigger than that with supplemental signals. The saturation headways with supplemental signals are $1.34 \mathrm{~s}$ for left turning vehicles and $1.39 \mathrm{~s}$ for through vehicles, while without supplemental signals are $1.42 \mathrm{~s}$ for left turning vehicles and $1.46 \mathrm{~s}$ for through vehicles. After installing supplemental signals, saturation headways and basic saturation headways both decreased whatever for left turning or through vehicles. Generally, the basic saturation headways are little bigger than saturation headways.

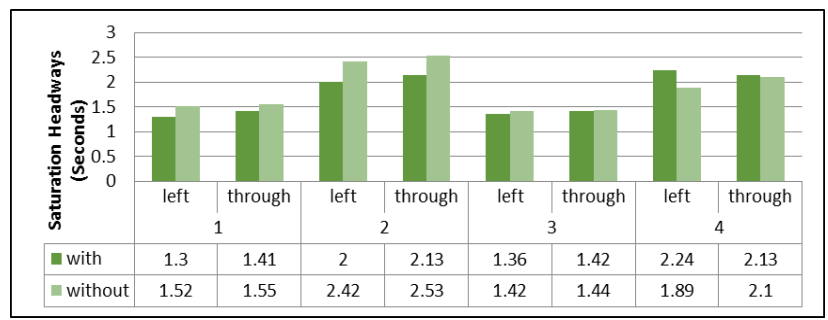

Figure 2. Comparison of the saturation headways.

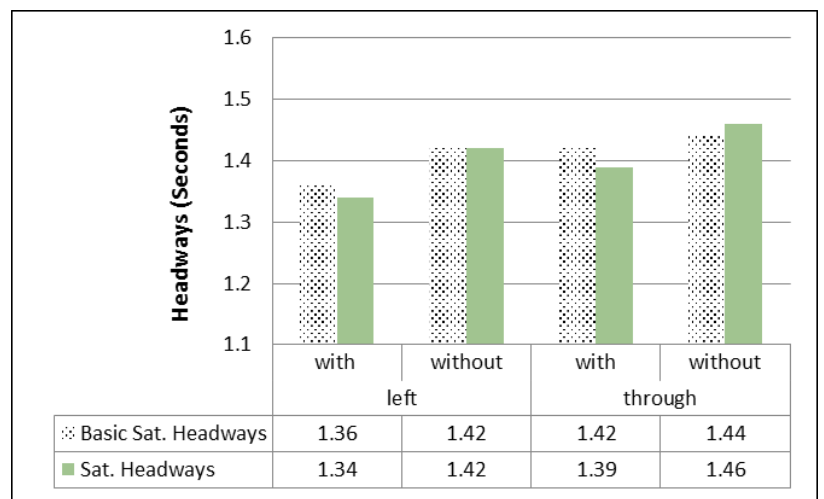

Figure 3. Comparison of saturation headways.

Summary of cycle numbers and means of SULT are presented in Table 3 . The SULTs in case 1 and case 3 are larger than that in case 2 and case 4 for both movements. Meanwhile, the SULTs in all cases with supplemental signals are smaller than that without supplemental signals. T-test was used to identify whether the mean values of SULT with supplemental signals were significantly different from that without supplemental signals. With a 95\% confidence level, the results showed that SULTs with supplemental signals were significantly different from those without supplemental signals statistically only in case 1 and case 3 . In case 2 and case 4 , the means of SULTs for left turning and through vehicles also decreased after installing supplemental signals, even though the results of t-test were not significant. In general, the supplemental signals were effective on decreasing SULT, especially when heavy vehicles existed in the first four vehicles.

Frequencies of the four cases were presented in Table 3. With the corrected method of SULT (Equation (3)) and collected data, SULTs at intersections with and without supplemental signals were calculated. For left turning vehicles, SULT with and without supplemental signals were 1.45 seconds and 1.60 seconds respectively. And for through vehicles, SULT with and without supplemental signals were 1.69 seconds and 2.10 seconds respectively.

Table 2. Summary of cycle number and mean values of SULT

\begin{tabular}{|c|c|c|c|c|c|c|c|}
\hline \multirow{3}{*}{ No. } & \multicolumn{7}{|c|}{ left turning } \\
\hline & \multicolumn{3}{|l|}{ with } & \multicolumn{3}{|c|}{ without } & \multirow[t]{2}{*}{$p$-value } \\
\hline & N. & Mean & Std. & $\mathrm{N}$ & Mean & Std. & \\
\hline case1 & 21 & 1.94 & 0.671 & 19 & 2.50 & 0.963 & 0.044 \\
\hline case2 & 31 & 1.08 & 1.057 & 36 & 1.16 & 0.746 & 0.700 \\
\hline case3 & 26 & 1.82 & 0.971 & 32 & 2.44 & 1.122 & 0.032 \\
\hline case4 & 36 & 1.23 & 0.883 & 84 & 1.27 & 1.142 & 0.056 \\
\hline sum & 114 & - & - & 171 & - & - & - \\
\hline \multirow{3}{*}{ No. } & \multicolumn{7}{|c|}{ through } \\
\hline & \multicolumn{3}{|l|}{ with } & \multicolumn{3}{|c|}{ without } & $p$-value \\
\hline & $\mathrm{N}$ & Mean & Std. & $\mathrm{N}$ & Mean & Std. & \\
\hline case1 & 47 & 2.19 & 1.572 & 32 & 2.95 & 1.267 & 0.025 \\
\hline case2 & 53 & 1.08 & 1.090 & 24 & 1.58 & 1.181 & 0.069 \\
\hline case3 & 35 & 2.19 & 0.945 & 19 & 2.77 & 1.092 & 0.047 \\
\hline case4 & 146 & 1.64 & 1.190 & 63 & 1.67 & 1.184 & 0.879 \\
\hline sum & 281 & - & - & 138 & - & - & - \\
\hline
\end{tabular}

\section{Conclusions}

The study measures the impacts of heavy vehicles and supplemental signals on SULT for both left-turning and through vehicles at signalized intersections. Saturation headways and SULT with supplemental signals are analysed. Based on data analysis, the following conclusions can be drawn:

1) The most saturation headways with supplemental signals are smaller than that without supplemental signals for both movements. The results of T-test also show that saturation headways with supplemental signals are significantly different from that without supplemental signals. The supplemental signals are effective to reduce the block of heavy vehicles. Meanwhile, it is not reasonable to ignore the block of heavy vehicles in traditional calculation of SULT.

2) At intersections with supplemental signals, SULTs are less than that at intersections only with primary signals, especially when heavy vehicles exist in the first four vehicles. The results of T-test also show that SULT with supplemental signals are significantly different from that without supplemental signals.

The study is only implemented in Nanjing, China. More samples from other areas should be included to test the conclusions. And also, more data should be collected to measure correction coefficients. In the study, only locations of heavy vehicles are included. If the number or proportion of heavy vehicles is also introduced, a better understand on effects of heavy vehicles may be obtained.

\section{Acknowledgement}

This study is funded by National Key Technology Support Program (No. 2014BAG01B06). The authors also would like to thank the graduate research assistants from the School of Transportation, Southeast University for their assistance in field study. 


\section{References}

1. Highway Capacity Manual. TRB, National Research Council, Washington, D.C. (2010)

2. Manual on Uniform Traffic Control Devices for Streets and Highways. U.S.Department of Transportation Federal Highway Administration. (2009)

3. H. Li, P.D. Prevedouros. Detailed observations of saturation headways and start-up lost times. Transportation Research Record (TRR)". TRB, National Research Council, Washington, D.C., (2002)

4. Highway Capacity Manual. TRB, National Research Council, Washington, D.C., (2000)

5. J. Bonneson, B. Nevers, J. Zegeer, T. Nguyen and T. Fong. Guidelines for quantifying the influence of area type and other factors on saturation flow rate. Technical Report. Texas Transportation Institute, College Station, Texas. (2005)

6. J.W. McMahon, J.P. Krane and A.P. Federico. Saturation flow rates by facility type. ITE Journal, $\mathbf{6 7}$, 46-50 (1997)

7. T. Limanond, P. Prabjabok, Kr. Tippayawong. Exploring impacts of countdown timers on traffic operations and driver behavior at a signalized intersection in Bangkok. Transport Policy. 17, 420427. (2010)

8. J. Joseph, G.L. Chang. Saturation flow rates and maximum critical lane volumes for planning applications in maryland. Journal of Transportation Engineering. 131(12), 2066-70. (2005)

9. R. Alyami, B.N. Janson. Effects of trucks on start-up lost time at signalized intersections. Transportation
Research Board Annual Meeting. Washington, D.C. (2010)

10. Survey of Motor Vehicle Use ABS (1972-2003). Series 9208.0, Australian Bureau of Statistics, Canberra, ACT. (2004)

11. X. Zhao, G. Ren. Analysis of vehicle headway characters at a signalized intersection. Journal of Transportation Engineering and Information. 8, 103108 (2010)

12. X. Yang, B. Zhuang, K. Li. Analysis of saturation flow rate and delay at signalized intersection. Journal of Tongji University (Natural Science). 34, 738-743 (2006)

13. S. Tsao, S. Chu. Adjustment factors for heavy vehicles at signalized intersections. Journal of Transportation Engineering, ASCE. 121(2), 150-157, (1995)

14. K. Kockelman, R. Shabih. Effect of vehicle type on capacity of signalized intersections. Journal of Transportation Engineering, ASCE. Vol. 126(6), 506-512 (2000)

15. E. Ramsay, J. Bunker, R. Troutbeck. Signalized intersection capacity reduction of trucks, In the Proceedings of the Fourth International Conference on Traffic and Transportation Studies. Science Press: Beijing, China. (2004)

16. P. Liu, J. Wan, W. Wang, Z. Li. Evaluating the impacts of an unconventional outside left-turn lane design on traffic operations at signalized intersections. Transportation Research Record (TRR). TRB, National Research Council, Washington, D.C. (2011) 